\title{
Acumulación de riqueza: sistemas agrarios en la región norte del departamento de La Libertad, El Salvador
}

\author{
Acumulação de riqueza: sistemas agrários na região norte do departamento "La Libertad", El Salvador
}

\author{
Carlos Ayala Duran \\ Lovois de Andrade Miguel
}

Universidade Federal do Rio Grande do Sul - UFRGS - Porto Alegre - Rio Grande do Sul - Brasil

\begin{abstract}
$\longrightarrow$
Resumo: O seguinte trabalho apresenta a dinâmica e a evolução dos sistemas agrários ao norte do departamento "La Libertad" em El Salvador. O mesmo baseia-se na teoria dos sistemas agrários, na versão francesa de Mazoyer y Roudart (1997 e 2010). Foram identificados quatro sistemas agrários: Indígena, colonial, cafetaleiro e liberal. No final do trabalho evidenciam-se as relações entre à agricultura e a acumulação de riqueza tanto em El Salvador como no norte do departamento La Libertad. Da mesma forma, é destacado como parte da indústria e do comercio possuem uma origem agrícola, particularmente derivada da acumulação de riqueza arrecadada nessa área. Finalmente, são discutidos alguns elementos que poderiam gerar uma crise no atual sistema agrário liberal.
\end{abstract}

Palavras-chave: Sistema agrário. El Salvador. La Libertad. Agricultura.

Abstract: The following article presents the evolution of the agrarian system from the northern department of La Libertad in El Salvador. The article is based on the theory of agrarian systems from the French school of Mazoyer e Roudart (1997 and 2010). Four agrarian systems were identified, namely: Indigenous, colonial, coffee-based and liberal. At the end of this work, remarks are made on accumulation of wealth in the whole country El Salvador, as well as in the studied region of La Libertad. Similarly, it's stressed how part of industrial and commercial sectors emerged from agriculture, particularly derived from the cited accumulation. Elements that may trigger the crisis of the current agrarian system are also part of final remarks.

Keywords: Agrarian systems. El Salvador. La libertad. agriculture. 


\section{Introducción}

El Salvador es un pequeño país de América Central, localizado en lo que coloquialmente se conoce como valle de las hamacas, por su alta actividad sísmica. El país ha tenido históricamente vocación agrícola. Desde el tiempo de la colonia, la explotación agrícola fue fundamental en sus relaciones con el imperio español. Ya en años más recientes, los temas agrarios, particularmente los relacionados con la reforma agraria, han tenido una importancia fundamental en el devenir del país, llegando al punto de generar grave inestabilidad política.

Pese a esta importancia histórica de la agricultura en El Salvador, la misma comenzará a perder peso como proporción del Producto Interno Bruto nacional desde la segunda mitad del siglo XX. Esto lleva aparejado el aumento de otros sectores de la economía, particularmente comercio y servicios (PRISMA, 2002). Resulta destacable el hecho que en El Salvador muchas empresas y conglomerados económicos de sectores como el industrial y servicios fueron fundados y consolidados gracias a un origen de capital proveniente de la agricultura, con lo que comprender las interrelaciones entre agricultura y los otros sectores se vuelve aún más importante para entender el devenir de algunos sectores industriales y comerciales. Para ello, se hace importante el estudio de la agricultura desde una perspectiva integradora y que pueda dar cuenta de su interrelación con otras esferas económicas. Con ello, para comprender el estado de la agricultura actual, es necesario estudiar las estructuras agrícolas que la precedieron, tanto a nivel de medio cultivado como del sistema social que la rodea. Es justamente en este momento que la teoría de sistemas agrarios se muestra particularmente valiosa. Para el caso particular de El Salvador, si bien existe interés por los temas agrícolas en el país, se evidencia un nivel de investigación relativamente bajo tanto desde las ciencias agrarias como desde algunas ciencias sociales, siendo prácticamente nulas investigaciones desde un enfoque de sistemas agrarios. Una notable excepción es el estudio del sistema agrario del distrito de riego y avenimiento de Zapotitán realizado por la Cooperación Técnica Francesa (CTF, 1988, 1988b); donde se aplicó una metodología similar a la teoría de sistemas agrarios.

Paralelamente, la elaboración de estudios agrarios desde una perspectiva parcelada y reduccionista es palpable, además de en El Salvador, en toda América Latina. Pese a ello, existen países como Brasil, y particularmente en el sureño estado de Rio Grande do Sul, donde el enfoque sistémico aplicado a la agricultura está ganando fuerza. Con ello, se han realizado estudios desde un abordaje sistémico en diversas localidades sulriograndenses de ese país, a saber: Maquiné (Kraemer y Miguel 2014), Campos Cima da Serra (Ambrosini, Miguel y Filippi, 2012), Tramandaí (Cotrim y Miguel, 2009), Encruzilhada (Ribas y Miguel, 2004), entre otras. De forma similar, Miguel (2009) hace una evolución de los sistemas agrarios en todo el Estado de Rio Grande do Sul. De forma paralela, La Universidade Regional do Noroeste do Estado do Rio Grande do Sul instituyó en 1988 un programa de investigación en "sistemas agrarios y desarrollo" (Silva et. al. 1997). Con todo ello, esta forma de abordar investigaciones sobre temas agrarios parece comenzar a probarse útil y ganar fuerza al menos en el sur de Brasil.

Ante esta falta de estudios agrarios en El Salvador sobre una perspectiva más integradora, fundamental para el desarrollo rural, el siguiente trabajo estudia el sistema agrario de la zona norte del departamento de La Libertad. La selección del área obedece a que la zona norte de dicho departamento presenta una unidad paisajística y climática uniforme catalogada como zona central de planicies de altura media y volcanes (USAID,2010), a que dicho departamento tiene una producción grande de granos básicos y particularmente de maíz (MINEC, 2009) y a que se encuentra localizada cerca de dos importantes centros urbanos: El de la capital del país y mayor ciudad del mismo, San Salvador, y el de la cabecera departamental de La Libertad, Nueva San Salvador (Santa Tecla). 
Por consiguiente, el resto del trabajo se divide de la siguiente forma: En el próximo capítulo se presenta la metodología utilizada, para entrar a continuación a la descripción actual del espacio agrario. En seguida, se describe la evolución y diferenciación de los cuatro sistemas agrarios identificados, dando finalmente paso a una sección de discusión y consideraciones finales.

\section{Metodología}

Para la realización del presente trabajo, se utiliza la teoría de sistemas agrarios propuesta por Mazoyer y Roudart (1997). Según ella, se puede entender como sistema agrario: "Un sistema de explotación del medio históricamente construido y durable, un conjunto de fuerzas de producción adaptado a las condiciones bioclimáticas de un espacio definido y respondiendo a las condiciones y necesidades de un cierto momento" (Mazoyer y Roudart, 1997 apud Miguel, 2009).

Así, se puede entender también como sistema agrario el instrumento intelectual que permite aprender la complejidad de toda forma de agricultura real, gracias al análisis metódico de su organización y funcionamiento; permitiendo representar las transformaciones incesantes de la agricultura en una región determinada del mundo como una sucesión de distintos sistemas (Mazoyer y Roudart, 2010). Con ello, concebir la agricultura practicada desde un enfoque de sistemas agrarios consiste en descomponerla en dos de sus subsistemas principales: El ecosistema cultivado y el sistema social productivo (Ibidem, 2010).

Teniendo este referente teórico como base para el estudio, se recolectó información bibliográfica tanto por medios físicos como electrónicos sobre el sistema agrario de la zona en cuestión. Dado que no existe información específica sobre sistemas agrarios en el área de estudio, se recopiló información proveniente de ciencias como agronomía, historia, biología, arqueología. De forma paralela se utilizó reportes oficiales y de organismos internacionales. Así mismo, se valió de información previa, conocimiento personal de la zona en cuestión y la información proporcionada por dos agrónomos en 2015 conocedores de la zona de estudio.

\section{Sistemas Agrarios}

\section{Descripción del Espacio Agrario}

El Salvador es un país de menos de 21,000 kms2, localizado en Centroamérica, con dos fronteras terrestres: al noroeste con Guatemala y al Norte con Honduras. Dividido en catorce departamentos, el Departamento de La Libertad tiene una extensión territorial de $1652.9 \mathrm{~km} 2$ con un total de 660,652 habitantes, dando una media de 400 habitantes por kilómetro cuadrado (MINEC, 2008). El país se encuentra rodeado por tres placas tectónicas: Norteamérica, Cocos y del Caribe. Debido a la influencia de esas placas tectónicas, el país, y la región centroamericana, tiene una actividad volcánica y sísmica intensa hecho fundamental para comprender la historia y la evolución de los sistemas agrarios tanto del país como del área delimitada de estudio.

En términos climáticos, el país tiene solamente dos estaciones: lluviosa y seca. La primera ocurre entre los meses de Mayo a Octubre, y la segunda de Noviembre a Abril. Si bien existen solamente dos estaciones en el año, se identifican también las llamadas transiciones seca-lluviosa (un mes entre abril a mayo) y una transición lluviosa-seca (alrededor de un mes entre octubre a noviembre) (CMPC, 2013). En el período 2010-2014, el país registró 1,784mm. de precipitación media anual (World Bank, 2015). De forma similar, El Salvador tiene un clima caliente con pocas oscilaciones en el año. La temperatura mínima anual oscila entre los $11-14{ }^{\circ} \mathrm{C}$ (zona norte y montañosa) hasta los $23-25^{\circ} \mathrm{C}$ en la zona costera (MARN, 2015). De forma similar, la temperatura máxima media anual puede alcanzar entre $18-23^{\circ} \mathrm{C}$ en la zona norte y $33-35^{\circ} \mathrm{C}$ en la zona costera y otras regiones calientes del país (Ibid, 2015).

Atendiendo a sus características ambientales y naturales, el país puede ser clasificado en tres zonas: 
Zona costera, zona central de planicies de altura media y volcanes y zona norte montañosa de tierras altas (USAID, 2010). Para el presente estudio fue seleccionada la zona central de planicies de altura media y volcanes del departamento de La Libertad, región que se ubica al norte de dicho departamento. La zona de estudio comprende de manera integral los municipios de San Pablo Tacachico, San Juán Opico, San Matías, Quezaltepeque, Ciudad Arce y Colón. Incluye de forma parcial los municipios de Sacacoyo, Talnique y Santa Tecla. Finalmente, abarca de forma marginal los municipios de Jayaque, Comasagua y Antiguo Cuscatlán. La región abarca aproximadamente $800 \mathrm{kms} 2$. La Imagen 1 muestra la representación de la división política del departamento y el área aproximada de estudio.

Imagen 1. Mapa del país y división Política del área de estudio (círculo rojo). Sombreado corresponde a la división municipal en el departamento de la Libertad y cómo dicho territorio fue abarcado de forma integral, parcial o marginal por el estudio. Fuente: Elaboración propia.

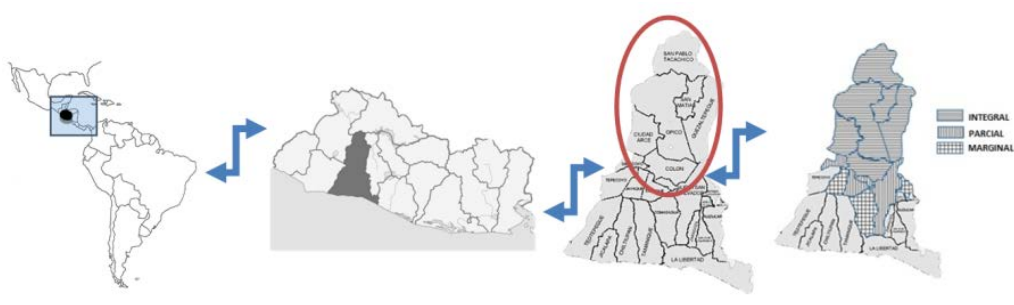

Los suelos del área de estudio son de origen volcánico. Desde un análisis pedológico, se tiene una porción importante de inceptisol (cerca del volcán de San Salvador y noreste), molisol (norte), Entisol, inceptisol, alfisol (noreste); alfisol, y ventisol.

La temperatura media anual del área de estudio puede oscilar entre $15-17^{\circ} \mathrm{C}$ la mínima y 27 $30^{\circ} \mathrm{C}$ la máxima, siendo relativamente menos caliente en las faldas del volcán de San Salvador. En ese sentido, según el Ministerio de Medio Ambiente y Recursos Naturales (MARN, 2015), la mayoría del área de estudio se cataloga desde el punto de vista climático como sabana tropical caliente, Sabana tropical calurosa o tierra templada (cerca de las faldas del volcán de San Salvador). Existe de la misma forma una parte ínfima de clima tropical de alturas, que corresponde con los puntos más altos del volcán de San Salvador (Ibid, 2015).

Según Adams (2007), el bioma actual del área de estudio (así como de todo el país) es bosque tropical. El bioma original, entre 22,000-14,000 años antes del presente, era sabana, tanto para el área de estudio como para todo el país y parte de la región centroamericana (Ibid, 2007).Una vez descrito el espacio agrario, se pretende presentar la dinámica y diferenciación de los sistemas agrarios sucedidos al norte de la Libertad.

\section{Sistema Agrario Indígena: 600- 1540 DC}

Este sistema agrario es el que mayor cobertura de tiempo tiene y probablemente del que menor cantidad de datos se posee, con lo que representa un reto adicional poder reconstruir la dinámica de este sistema agrario. De forma preliminar, según estimaciones de Mayozert y Roudart (2010), existe evidencia de la domesticación de la agricultura en la región de Mesoamérica aproximadamente entre 9000 a 4000 años antes del presente, particularmente en la parte sur de México. No obstante, en el área de estudio del norte de La Libertad, se tiene evidencia de que aproximadamente 600 años D.C. ya existía agricultura domesticada (MINED, 2008 y White, 2008). Debido a la naturaleza volcánica del país, existieron numerosas erupciones a lo largo de la historia y, gracias a excavaciones arqueológicas, se ha logrado identificar que ya para el año 600 D.C. en la zona de Cerén, Municipio de San Juán Opico, ya existían cultivos de maíz, frijol y calabaza (White, 2008; MINED, 2008) base fundamental de la dieta de los salvadoreños hasta la actualidad. La producción de alimentos en la zona de estudio se concentró en esos granos básicos, ya que tienen un carácter nutricional destacado y aseguraban el autoconsumo de los agricultores. De la misma forma, según White (2008), existe evidencia del cultivo de cacao (si bien residual, a diferencia de otras zonas del país) así como la extracción de miel y cera. Fueron encontradas también huertas cerca de las casas, 
cultivos en línea en terrenos bien drenados; no obstante no fue encontrada evidencia de irrigación de los mismos, lo que indicaría que la agricultura dependía fundamentalmente del agua lluvia. Similarmente, existe evidencia de la utilización en Cerén de estiércol humano como fertilizante (Ibid, 2008), indicando que para esta época la utilización de este fertilizante ya era una práctica conocida. En sentido parecido, Mckee 1990 y 1996 apud Miller, 2002; apuntan que existe indicios de la utilización de desechos o rubbish como fertilizante. Como es de esperarse, a esta altura de la historia la evidencia indica que la agricultura estaba basada en la práctica derribada y quemada (MINED, 2008 y White, 2008). De forma similar, existe evidencia indicando que ya existía una rotación de cultivos y que una parte de la agricultura donde se producían alimentos para consumo propio era realizada en los solares de las casas (MINED, 2008). Según Dixon, 2011 y Sheets et. al., 2012 entre otros, existe evidencia de otros cultivos menos tradicionales como la yuca, malanga o la cebadilla. Nuevas investigaciones indican que algunos de estos, la yuca por ejemplo, tenían gran importancia para los antiguos pobladores en el norte de la libertad (Sheets et. al. 2011 y Sheets et. al. 2012).

En sentido similar, fueron encontrados algunos instrumentos manuales para el cultivo, como hacha de piedra, puntas de proyectil para cazar, raspadores de piel y molinos de piedra (MINED, 2008). Todo indica que estos instrumentos eran utilizados de forma más o menos regular en el área de estudio. De forma excepcional, en excavaciones recientes, fue encontrada una hacha de Jade, procedente de Motagua, Guatemala (Sheets et. al 2006). Con ello, se hace posible considerar la existencia de intercambios de instrumentos para la agricultura (entre otros) con poblaciones de Guatemala. En forma paralela, no se ha encontrado evidencia de la utilización de metales en esta localidad en este sistema agrario, si bien es posible que existieran también intercambios con poblaciones aztecas (además de con pobladores de Guatemala), lo que permitiría la explotación agrícola con instrumentos metálicos como el oro o la plata en el área de estudio. De forma similar, existe evidencia de creación de piezas de cerámica para guardar y servir alimentos principalmente (MINED, 2008 y Sheets, 2013), así como de la creación de tejidos (MINED, 2008).

La fuerza de trabajo puede ser dividida en éste sistema agrario mediante dos épocas y pueblos originarios diferentes: Los mayas en un primer momento hasta alrededor del año 1000, y los pipiles hasta el momento de la conquista española. De la fuerza de trabajo en la era Maya existe poca evidencia que retrate de forma clara como se organizaba la producción agrícola en el área de estudio. No obstante, todo indica que la estratificación social y la composición de la fuerza de trabajo en otras ciudades importantes del imperio maya es la misma que se desarrolló en el norte de la libertad hasta aproximadamente el año 1000.Si bien se cree que no existía en todo el imperio maya un principio organizador de la sociedad, y en este caso de la producción agrícola, al ser una sociedad no igualitaria, la fuerza de trabajo era estratificada en torno a las figura de: Líder o soberano, nobles y sacerdotes, agricultores (plebeyos o comuneros) y esclavos (Morley y Brainerd,1983). En lo que atañe a la agricultura, los agricultores o comuneros eran los que desarrollaban la mayor parte del trabajo en las parcelas agrícolas a cargo del noble, quién se aseguraba que se pagase tributo al líder o soberano (Ibid, 1983). De la misma forma, los esclavos por su condición subordinada, eran obligados a trabajar en cualquier tipo de tareas, incluyendo la agricultura.

Posteriormente, y después del colapso de la civilización maya, los indios pipiles poblaron el área de estudio. Pipil significa en nahuatl noble, señor o príncipe; lo que ya sugiere que la estructura de la sociedad era parecida a la maya. Como lo recoge diferentes fuentes (White, 2008 y MINED, 2008), la sociedad pipil estaba cimentada en torno al cacicazo. En consecuencia, la sociedad se estructuraba según las categorías: Líder o rey, Noble, Comunero y Esclavos (White, 2008 y MINED, 2008). Dentro de los nobles pipiles estaban incluidos el clero, políticos y los guerreros que habían ganado reconocimiento. 
Originalmente, era encomendado a ellos la explotación de la tierra, si bien no la trabajaban de forma directa. Los llamados comuneros aglutinaban a la mayoría de la población pipil, la que carecía generalmente de mayores privilegios y la que cultivaba la tierra que era asignada a los nobles. Finalmente los esclavos representaban el último escalafón en la estructura social, quienes eran obligados a trabajar en la tierra y muchas veces eran sujetos de sacrificios.

Siendo este sistema agrario el más amplio de los cuatro identificados, el modo de acceso a la tierra tiene algunas características particulares en función del momento histórico en particular. Originalmente, se cree que los pobladores eran cazadores y colectores, pasando posteriormente a una fase agrícola domesticada alrededor del año 600 D.C. En este primer período hasta el año 600 D.C, se cree que el acceso a la tierra era libre. Posteriormente, tanto en la época maya como durante el dominio pipil, el acceso a la tierra era más restringido. Durante la época Maya, la tierra era asignada a nobles quienes se aseguraran que los comuneros y esclavos la trabajasen y pagase el tributo al líder o rey (Morley y Brainerd, 1983). Posteriormente, en la era pipil alrededor del año 1000 D.C., el modo de acceso a la tierra era parecido, al ser controlado por la elite pipil gobernante, que asignaba su uso (MINED, 2008). Los nobles administraban la tierra y tenían para trabajarla los llamados comunes y esclavos, quienes debían pagar tributo (Ibid, 2008).

En el tema de las relaciones de producción e intercambio, existe evidencia que había intercambio de maíz, algodón y algunos tejidos con los pipiles de Izalco, mismos que a su vez aportaban cacao, ya que dicho fruto no era común en La Libertad (White, 2008). De forma similar, ya en el primer sistema agrario se identificó la producción de añil, si bien la misma era residual, situación que iría a cambiar en el próximo sistema agrario. Como se ha mencionado anteriormente, todo lleva a creer que existían intercambios entre los pipiles de todo el país y del norte de la Libertad con poblaciones aztecas y guatemaltecas.
Como es de esperarse, este sistema agrario entrará en una crisis y colapso como fruto de la conquista por parte del imperio español, lo que iba a trastornar la forma de practicar agricultura, producción de excedentes, número de población local y ocupación de los mismos.

\section{Sistema Agrario Colonial 1540-1881}

La conquista de El Salvador por parte del imperio español hizo mudar de forma marcada el sistema agrícola salvadoreño. En un territorio pequeño y relativamente pobre en comparación con otras colonias españolas en América, era necesario explotar recursos rentables para la corona ibérica. Este recurso rentable se vio materializado con el llamado oro azul: el añil. Probablemente la principal fuente de explotación económica en todo el país era la producción de añil, índigo o Indigofera suffruticosa, cuya planta se conocía localmente como xiquilite. El mismo era utilizado principalmente como colorante para tejidos, muy codiciados en el siglo XVI en Europa. El manual de historia del ministerio de educación recoge la importancia de este producto de forma poéticamente decadente: "La vida colonial es apenas imaginable sin este tinte, maravilloso tanto para los teñidores europeos como para los productores salvadoreños". (MINED, 2008: p. 78).

El área de estudio tuvo importante producción añilera, siendo un excedente sustancial que era exportado a España para su posterior comercio en el viejo continente. Existían otros excedentes agrícolas relevantes como el bálsamo, algodón, tejidos, maíz, y algunas hortalizas; mismos que incluso en algunas ocasiones eran aceptados como pago en concepto de tributo (White, 2008 y MINED, 2008). La utilización de estos productos como pago de tributo puede ser explicado, al menos parcialmente, debido a que en el territorio nacional, y en el área de estudio en particular, no existía mayor explotación de oro u otros metales preciosos que eran altamente valorados por la corona española. En un sentido similar, se llegó a pagar tributo con trabajo indígena, algo observado en otras colonias en América. 
Existió por su vez, una pequeña extracción marginal de hierro en Quezaltepeque, si bien la disponibilidad de la explotación del recurso era limitada, lo que generó su posterior abandono (White, 2008). Asimismo, desde mediados del siglo XIX, el café comenzará a ser cultivado en el país marginalmente, ya que la poca disponibilidad de tierra apta para su cultivo y la forma de tenencia de la misma no dejarían despuntar su producción (MINED, 2008).

Por otra parte, después de la pacificación de la región producto de la conquista europea, los españoles traen y comienzan a criar ganado ibérico, mismo que sería integrado en la producción de añil, principalmente para desmatar de áreas y para la producción marginal de ganado de corte. De la misma forma, el ganado era utilizado para la producción de cuero así como para engrasar las carrozas que transportaban el xiquilite (MINED, 2008). Este ganado supuso un grave problema para las poblaciones locales ya que los mismos degradaron de forma notable el ecosistema de los pobladores originarios, llegando a comer cosechas utilizadas para el consumo (White, 2008); lo que propició incluso en un momento la promulgación de una ley por la que se permitía matar a ganado que estuviera en propiedad indígena.

De forma similar, los españoles introdujeron un nuevo arsenal de instrumentos y equipamientos para la agricultura: Machetes, palas y arados de hierro por ejemplo (Martínez, 2002). En ese sentido, los conquistadores ibéricos trajeron consigo otros metales como el hierro, el cobre y el plomo; que serían incorporados de forma marginal en la agricultura (MINED, 2008).

La fuerza de trabajo en el sistema agrícola del norte de la libertad era básicamente indígena pipil. Originalmente, se identificó que la producción de añil tenía efectos perjudiciales en la salud, lo que motivó la promulgación de leyes por parte de la corona española prohibiendo en dos ocasiones diferentes, la utilización de la mano de obra indígena en la producción de añil (Solórzano, 1982), mismas que no fueron respetadas (White, 2008). Similarmente, enfermedades, jornadas laborales extenuantes, enfermedades y degradación de bioma y medios de vida por parte del ganado, generó una gran disminución de la población indígena originaria. Esta población indígena a su vez fue obligada a pagar tributo, a lo que aparentemente ya estaban acostumbrados desde la época del cacicazo pipil (MINED, 2008). A esta fuerza de trabajo se le adicionaría la población esclava, negra principalmente, traída de África por los españoles.

En ese mismo sentido, en este sistema agrario pueden distinguirse algunas categorías sociales bastante marcadas. Una de ellas estaba compuesta por los conquistadores españoles, si bien posteriormente vendrán otros colonos de ese país a instalarse en la región. Otra categoría de gran importancia eran los indígenas principalmente pipiles, con su propia estratificación social. De forma residual, los españoles trajeron esclavos, principalmente africanos, para suplir la bajada en la fuerza de trabajo indígena y las prohibiciones de la corona española a que los indios explotaran la producción de añil. (MINED, 2008). Posteriormente, y en virtud de la mezcla entre razas, surgirán los mestizos. Por otra parte, y dependiendo del momento histórico en particular, la elite gobernante española utilizó alguna forma de explotación de la tierra y de la mano de obra indígena que podía ser encomienda (MINED, 2008), hacienda (Alvear, 2002) o cualquier esquema que permitiera la explotación del medio y de la mano de obra, mismas que rozaban con la esclavitud, si bien suele decirse falazmente que dicha práctica no existió de forma generalizada en el país ni en el norte de la libertad.

El sistema de acceso a la tierra en el sistema agrario colonial era diverso. Por ejemplo, existía un número reducido de tierras ejidales que eran de propiedad de la municipalidad y en la que se trabajaba de forma comunal (White, 2008 y Lauria, 1999). Existían de la misma forma tierras comunales y tierras al servicio de la explotación española. Las tierras comunales eran de todos y eran de igual forma trabajada en su conjunto, las mismas tenían un gran arraigo cultural indígena, al ser trabajadas 
principalmente por ellos en base a sus costumbres, dietas y aspiraciones. La comprensión del acceso a la tierra será fundamental para dilucidar el cambio del sistema agrario colonial al siguiente.

Este sistema agrario se iría a agotar poco a poco, hasta llegar a un punto de colapso y quiebre claro. Inicialmente, existió una crisis en la producción de añil al inicio del siglo XIX, que tardó casi 20 años en recuperarse (Lauria, 1999). Así mismo, la corona española estaba bastante mermada por las guerras napoleónicas en Europa, lo que redujo su influencia en América en general y en El Salvador en particular. Adicionalmente, existió una creciente competencia en la producción de añil de India y Venezuela, y se comenzó a producir los colorantes sintéticos, que prácticamente hizo que se desplomaran los precios del añil a nivel internacional (White, 2008); haciendolo poco atractivo para continuar siendo explotado en El Salvador.

En ese sentido, en un país "pobre", sin la presencia de minas de metales $u$ otro recurso apetecible en el mercado internacional, se necesitaba encontrar algún producto con gran valor que fuera sujeto a comercio. Poco a poco, el café irá a implantarse como principal producto de exportación nacional, como lo haría en otras latitudes latinoamericanas; si bien la expansión del mismo se veía supeditada a la forma de tenencia de la tierra en El Salvador y en el norte de la libertad. Como mencionado, la misma estaba estructurada en tierras comunales, ejidales y propiedades privadas. Dado que la tierra para producir café necesita unos requerimientos particulares, como temperatura templada o una elevación media sobre el nivel del mar, y que algunas tierras aptas eran comunales o ejidales, el sistema de tenencia de la tierra en todo el país se consideraba inadecuado. Por ello, en 1881 se promulga por decreto de ley la abolición de las tierras comunales y ejidales. Esto tendrá un marcado impacto tanto en el área de estudio como en todo el país, y es exactamente éste el punto de quiebre para el tercer sistema agrario identificado.

\section{Sistema Agrario Cafetalero 1885-1960}

La producción de café fue poco a poco substituyendo al añil como principal producto de exportación en todo el país y en La Libertad en particular. Se puede ver la evolución de la producción de todo el país en quintales en parte de los siglos XIX y XX en el cuadro 1. Pese a que el café comenzaba a tener paulatinamente mayor importancia en relación al añil, se tenía el inconveniente que mucha de las tierras óptimas para ese cultivo eran tierras comunales o ejidales, mismas que eran trabajadas de forma conjunta y sin tener derechos de propiedad claramente definidos. Por ello, se suele argumentar que la abolición de dichas formas de tenencia de la tierra en 1881, estuvo grandemente influenciada por el incipiente desarrollo cafetalero del país.

Con la abolición de tierras ejidales y comunales, en primer momento ocurrirá un proceso de repartición de pequeñas propiedades a los antiguos trabajadores. No obstante, muchas veces esas pequeñas propiedades fueron adquiridas agricultores grandes, ya sea por medio de venta, intimidación, ocupación, pérdida por no acatar disposiciones o por usurpación (Portillo, 2002); dando lugar a una concentración de tierras por parte de grandes propietarios, lo que generarán latifundios y haciendas por un lado (Lindo, 2011); y numerosas pequeñas propiedades por otro. Siendo así, el acceso a la tierra era principalmente de forma individual, con título de propiedad regularizada.

Todo parece indicar que este sistema agrario ha servido como origen de las grandes concentraciones de tierra en el país. Como se verá más adelante, para el año 1961, seis familias salvadoreñas aglutinaban 71,923 hectáreas (Burke, 1976). De la misma forma, este aglutinamiento de tierras es considerado crucial en la llamada acumulación originaria de capital en El Salvador (Menjívar, 1980). 
Cuadro 1. Producción y exportación de Café, 18601940. Fuente: MINED,2008b.

\begin{tabular}{|c|c|c|c|}
\hline ño & $\begin{array}{l}\text { Producción } \\
\text { (quintales) }\end{array}$ & $\begin{array}{l}\text { Exportación } \\
\text { (quintales) }\end{array}$ & $\begin{array}{l}\text { Estimado de tierras } \\
\text { bajo cultivo de café } \\
\text { (hectáreas) }\end{array}$ \\
\hline 860 & 11,000 & --- & +-- \\
\hline 880 & 200,000 & 166,000 & --- \\
\hline 885 & 300,000 & --- & --- \\
\hline 890 & 600,000 & --- & --- \\
\hline 901 & 556,000 & 437,326 & 42,000 \\
\hline 905 & 775,000 & 620,391 & 60,000 \\
\hline 910 & 772,000 & 618,347 & 59,000 \\
\hline 916 & 988,674 & 777,326 & 61,000 \\
\hline 920 & $1,026,715$ & 817,108 & 73,000 \\
\hline 924 & $1,304,347$ & $1,061,065$ & 80,000 \\
\hline 929 & $1,413,492$ & $1,017,021$ & 98,000 \\
\hline 932 & $1,357,321$ & 862,065 & 97,000 \\
\hline 933 & $1,204,434$ & 100,000 & --- \\
\hline 934 & $1,332,224$ & $1,084,478$ & 104,000 \\
\hline 935 & $1,245,900$ & $1,088,413$ & 106,000 \\
\hline 936 & $1,559,819$ & $1,074,108$ & 109,000 \\
\hline 937 & $1,466,552$ & $1,469,956$ & --- \\
\hline 938 & $1,386,982$ & $1,169,652$ & 107,000 \\
\hline 939 & $1,455,577$ & $1,212,869$ & 100,000 \\
\hline 940 & $1,266,863$ & $1,231,500$ & 91,000 \\
\hline
\end{tabular}

El café llegó a tener tal importancia en el país entero, que se calcula que dicho grano contabilizó el $90 \%$ de las ganancias producto de la exportación antes de la Gran Depresión de 1929 (Ching, 2011). De forma similar, hasta el inicio de los gobiernos dictatoriales militares en el país en 1929, todos los presidentes en el siglo $X X$ provenían del sector cafetalero. En este sentido, con vías a la exportación del grano, se desarrolló infraestructura de gran calado, particularmente carreteras y el ferrocarril que atraviesa de forma central el departamento de La Libertad, y de forma marginal la zona de estudio.

La mano de obra en este sistema agrario suele denominarse como libre (Ibid, 2011), si bien existían diversas restricciones a la venta de fuerza de trabajo. Por ejemplo, existían leyes contra la vagancia (Menjivar, 1980 y Lindo, 2011) las cuales obligaban prácticamente a los agricultores a trabajar en haciendas; o en su defecto a tener oficio conocido.
En el modo de explotación del medio se tenían diversas prácticas. Por ejemplo, la producción de azúcar por parte de los ingenios fomentaba la quema de la caña verde, para poder cortarla con mayor facilidad. Esta práctica agrícola relativa al cultivo de caña, es incluso persistente hasta la actualidad. Por otra parte, al menos desde este momento ya hay indicios de prácticas agrícolas dándole descanso a la tierra e incluso utilizando técnicas de barbecho.

Si bien no existen categorías sociales definidas como tal, es innegable que existían por ejemplo grandes productores de café principalmente, agricultores de subsistencia; así como jornaleros y colonos (MINED, 2008).

Las relaciones de producción giraban en torno al excedente de café cultivado. El mismo era destinado originalmente para las exportaciones. Otros productos como el bálsamo y el azúcar continuaban siendo exportados con menor importancia relativa. De forma paralela, en el área de estudio se continuó cultivando granos básicos para consumo, e incluso, para intercambio y comercio, así como yuca, hortalizas y azúcar (OEA, 1977).

Finalmente, este sistema agrario vería su fin por diversos motivos. Poco a poco la importancia del café fue disminuyendo debido a la disminución de su precio internacional, en virtud del crack de 1929 y su subsiguiente crisis económica. Posteriormente, en los años cincuenta, se comenzó a dar un impulso mayor a semillas híbridas y fungicidas de origen no orgánico. $Y$ es este el punto rompimiento con el sistema agrario cafetalero, que dará paso sistema agrario de corte liberal.

\section{Sistema Agrario Liberal 1960- actualidad}

El último sistema agrario identificado ha sido llamado de Liberal, mismo que tendrá dos características: La aplicación tropicalizada de la revolución verde y una disminución de la importancia del sector agrícola en general.

El paquete tecnológico de la revolución verde comenzó a utilizarse en El Salvador alrededor de los años 50, fuertemente impulsado por las instituciones 
estatales. Debido a la cercanía con la capital, todo indica que el mismo se comenzó a utilizar en el área de estudio a finales de la década de los sesenta e inicios de los setenta, si bien de forma incipiente. No obstante, la penetración de ese paquete tecnológico tiene características especiales en el área de estudio, siendo que la misma se basó en la alta utilización de insumos agroquímicos y semillas no criollas. En ese sentido, actualmente existe una alta penetración de semilla híbrida en la producción de maíz. Como lo muestra el cuadro 2, se contabiliza que el $96 \%$ de la superficie sembrada de maíz en el departamento de la Libertad es semilla híbrida (MINEC, 2009). Paralelamente, alrededor de un $89 \%$ de los productores en todo el departamento aplican control químico de plagas (Ibid,2009). De forma antagónica, la moto mecanización no impactó tanto la producción agrícola del área de estudio. Siendo así, el último centro agrícola reporta la utilización de 1394 tractores en todo el departamento de La Libertad para la cosecha 2007-2008 (Ibid, 2009). Eso puede verse explicado, al menos de forma parcial, por el hecho que alrededor del $90 \%$ de las propiedades en el departamento tienen una extensión de hasta 2 hectáreas (Ibid, 2009); con lo que se hace muy difícil que esos productores puedan tener la capacidad económica para adquirir un equipo tan costoso o incluso que el mismo sea deseado dado el tamaño de las propiedades.

De la misma forma, todo el departamento muestra un comportamiento similar en lo que adquisición de insumos más costosos se refiere: Existen solamente 1532 arados de hierro y 97 sembradoras mecánicas; del total de 39247 (MINEC,2009).

Cuadro 2: Relación de área cultivada y área cultivada con semilla híbrida de maíz. Elaboración propia en base a MINEC, 2009.

\begin{tabular}{|l|l|l|l|}
\hline Municipio & $\begin{array}{l}\text { Área } \\
\text { Cultivada } \\
\text { (Manzana) } \\
\text { (0,69 ha) }\end{array}$ & $\begin{array}{l}\text { Área } \\
\text { Cultivada } \\
\text { semilla } \\
\text { híbrida }\end{array}$ & Porcentaje \\
\hline $\begin{array}{l}\text { San Pablo } \\
\text { Tacachico }\end{array}$ & $2,707.83$ & $2,622.32$ & $96.84 \%$ \\
\hline San Matías & $1,834.55$ & $1,823.04$ & $99 \%$ \\
\hline San Juán Opico & $5,870.29$ & $5,709.49$ & $97.26 \%$ \\
\hline Quezaltepeque & $1,821.60$ & $1,803.21$ & $98.99 \%$ \\
\hline Ciudad Arce & $4,336.12$ & $3,852.08$ & $88.83 \%$ \\
\hline Sacacoyo & 838.30 & 810.68 & $96.70 \%$ \\
\hline Talnique & 403.49 & 381.90 & $94.64 \%$ \\
\hline Santa Tecla & 765.76 & 752.02 & $98.20 \%$ \\
\hline Antiguo Cuscatlán & 154.42 & 154.29 & $99 \%$ \\
\hline SUBTOTAL & & & $99.64 \%$ \\
\hline Total departamento & $32,317.11$ & $31,160.41$ & $96.42 \%$ \\
\hline
\end{tabular}

En éste período de tiempo, El Salvador se ha caracterizado por ser exportador de algunos productos agrícolas tradicionales (Rosa, 2005); como el café o la azúcar. No existe información pormenorizada del área de estudio sobre relaciones de intercambio. Sin embargo, como lo señalan indicadores macroeconómicos de exportación, existen producciones de azúcar y café provenientes de la zona de estudio que son exportados hacia el extranjero.

En relación a la segunda característica de este sistema agrario se evidencia una disminución de la importancia del sector agrícola en general. Desde 1970 y hasta el año 2000, el PIB agrícola ha pasado de representar del 19\% al 12\% del PIB total del país (PRISMA, 2002). Similarmente, en ese mismo período de tiempo, el porcentaje de préstamos que el sector agrícola recibía en relación a los otros sectores económicos pasó de $27 \%$ al $7 \%$, en favor del sector comercio (Ibid, 2002). Por otra parte, en 1978, el 81\% de la recepción de divisas en el país era gracias al sector agroexportador tradicional, alcanzando solamente un 11\% en el año 2000 (Ibid, 2002). Esta fuente de divisas vino a ser substituidas en parte por las remesas enviadas por compatriotas viviendo en el exterior, mismas que oscilan entre el $12-17 \%$ del PIB salvadoreño. Simultáneamente, el porcentaje de empleos en agricultura en relación al total de empleos en la economía se redujo entre 1994 (28\%) y el año 2012 (21\%) en un 7\% (Ibid, 2002).

Junto con estos cambios macroeconómicos en la agricultura, se evidencia que el presupuesto que la administración pública central destina a la agricultura ha venido reduciéndose desde los años ochenta. En ese sentido, el porcentaje del presupuesto agrícola 
como proporción del presupuesto público llegó a 1,4\% en el período 2006-2010 (Redes,2014), uno de los más bajos en Centroamérica, como lo muestra el cuadro 3.

Cuadro 3: Participación del presupuesto agrícola en el presupuesto público en Centroamérica. Fuente: REDES, 2014.

\begin{tabular}{|l|l|l|l|l|l|l|}
\hline País/Período & $\begin{array}{l}\mathbf{9 8 0 -} \\
\mathbf{1 9 8 5}\end{array}$ & $\begin{array}{l}\mathbf{9 8 6 -} \\
\mathbf{1 9 9 0}\end{array}$ & $\begin{array}{l}\mathbf{9 9 1 -} \\
\mathbf{1 9 9 5}\end{array}$ & $\begin{array}{l}\mathbf{9 9 6 -} \\
\mathbf{2 0 0 0}\end{array}$ & $\begin{array}{l}\mathbf{0 0 1 -} \\
\mathbf{2 0 0 5}\end{array}$ & $\begin{array}{l}\mathbf{0 0 6 -} \\
\mathbf{2 0 1 0}\end{array}$ \\
\hline Costa Rica & $4.0 \%$ & $0.4 \%$ & $.1 \%$ & $.8 \%$ & $.7 \%$ & $.8 \%$ \\
\hline El Salvador & $.4 \%$ & $.1 \%$ & $.5 \%$ & $.9 \%$ & $.3 \%$ & $.4 \%$ \\
\hline Guatemala & $.2 \%$ & $.1 \%$ & $.9 \%$ & $.0 \%$ & $.6 \%$ & $.3 \%$ \\
\hline Honduras & $8.7 \%$ & $3.8 \%$ & $.2 \%$ & $.1 \%$ & $.1 \%$ & $.5 \%$ \\
\hline Nicaragua & $.6 \%$ & $.0 \%$ & $.1 \%$ & $.1 \%$ & $.8 \%$ & $.0 \%$ \\
\hline Panamá & $.6 \%$ & $.8 \%$ & $.1 \%$ & $.4 \%$ & $.2 \%$ & $.3 \%$ \\
\hline
\end{tabular}

Las principales actividades agroflorestales de transformación siguen teniendo como base en este último sistema agrario la producción de granos básicos principalmente para autoconsumo y eventualmente para comercialización. De la misma forma, se produce café e incluso existían ingenios azucareros (MINEC, 2009). Existe de la misma forma producción de hortalizas y frutas principalmente autoconsumo o intercambio, de forma destacada en el distrito de riego en el valle de Zapotitán. De forma mucho más minoritaria, existe creación de ganado, bovino, porcino y aves (Ibid, 2009)

Los equipamientos agrícolas van a sufrir un cambio dramático. Como se ha mencionado, desde los años sesenta comenzará a penetrar de forma mucho más agresiva las semillas híbridas y los fertilizantes, herbicidas y otros insumos de origen no orgánico, marcadamente químico. En ese sentido, se aprueba en 1971 la Ley de Certificación de Semillas y Plantas y dos años después la Ley obre Control de Pesticidas, Fertilizantes y Productos para Uso Agropecuario. Esas leyes tenían como claro objetivo promover el paquete tecnológico de la revolución verde.

Sobre el referido tema, empresas estadounidenses y algunas europeas tuvieron también un papel fundamental en la asesoría técnica y promoción de esa nueva tecnología. Consecuentemente, ya en 1974 casi el 40\% de los fertilizantes utilizados en el país eran estadounidenses, y $32.5 \%$ eran alemanes, seguidos por los de origen holandés; dando una media de utilización de 0.21 toneladas métricas por hectárea de tierra arable (USAID, 1975). Un reporte actual sugiere que la tendencia se mantiene inalterada (MAG, s.d).

La fuerza de trabajo prácticamente no sufrió ningún cambio. Se mantuvieron pequeños agricultores, medios y grandes propietarios privados (comerciales e individuales) y jornaleros que trabajan en propiedades grandes. Como categorías sociales, se puede hablar que existe un número reducido de grandes productores y muchos pequeños agricultores; herencia del sistema agrario anterior. En ese sentido, desde la década de los setenta, y como producto de una concentración en el sistema agrario cafetalero, se identifica la existencia de una concentración de tierra marcada. Para esa misma década, se calcula que más del $80 \%$ de las propiedades de todo el departamento de la libertad eran minifundistas, con una tendencia similar en el resto del país (OEA, 1977). Datos más recientes, específicamente de 2013, apuntan en la misma dirección, indicando que en el mismo departamento, el $89 \%$ de las propiedades tienen hasta 2 hectáreas (MAG, 2014).

En este sistema agrario se ha practicado una combinación de algunas técnicas de cultivo. Por ejemplo, se continúa utilizando para la zafra de la caña la quema, lo que hace más fácil su corte. Similarmente, en otras partes se cultiva dejando la tierra descansar e incluso se utiliza técnica de barbecho. Si bien existen algunas áreas de forraje (MINEC,2009), la misma es un porcentaje mínimo, incluso casi despreciable.

Por otra parte, el acceso a la tierra es muy parecido al sistema agrario anterior, existiendo una tendencia hacia el acceso individual con título de propiedad de productor. Pese a ello, persisten algunas otras formas como las cooperativas o incluso las no definidas que incluyen préstamos de tierras (MINEC, 2009); si bien las mismas son marginales. 


\section{Discusión: Acumulación de Riqueza}

Como ha podido quedar de manifiesto, la evolución de los sistemas agrarios al norte de La Libertad sigue una trayectoria relativamente estable. La región ha sido originalmente productora de alimentos para autoconsumo, marcadamente granos básicos y otras hortalizas, mientras se tenían algunos otros productos particulares para su explotación, ya sea añil, café, azúcar, etc. De la misma forma, la lógica de la evolución del sistema agrario ha estado directamente vinculado a los acontecimientos políticos y económicos de la elite gobernante del país, y dado su cercanía con la capital, los cambios en esta región han venido usualmente generados de forma paralela con los cambios acontecidos en la capital e incluso en todo el país: conquista, formas de tenencia de la tierra, utilización de nueva tecnología, etc.

Cabe señalar que el sistema agrícola cafetalero sirvió de base para una acumulación y concentración de riqueza en el país por un lado y para definir la estructura de la posesión de tierras por otro. Desde la abolición de las tierras comunales y ejidales, un grupo reducido de familias como los Dueñas, Guirola, Samayoa (MINED,2008), Álvarez Lalinde y Deninger (Portillo, 2002) entre otras, han logrado acumular grandes cantidades de tierra. Burke (1976) calculaba en ese sentido que para el año 1961 un reducido número de 6 familias (Guirola, Sol, Dueñas, Daglio, Samayoa y Romero Bosque) concentraban una proporción de 71,923 hectáreas en todo el país. Para el caso particular de la zona de estudio, la familia Dueñas concentraba ya en el período 1987- 1901 no menos de 6,908 hectáreas de terrenos, dividido en tan sólo tres municipios: Colón, Opico y Santa Tecla (Portillo, 2002). Por su parte, la familia Guirola, por medio de la Sociedad Agrícola Ángel Guirola - Antonio Martínez atesoró en ese período fincas por un total de 133 hectáreas en la zona de estudio en el mismo quinquenio (Ibid, 2002). Similarmente, la familia Álvarez Lalinde ya acumulaba propiedades por un total de 4,147 hectáreas; con un importante porcentaje de propiedades destinadas a la explotación de café.
En contraposición con estas familias que contaban con grandes extensiones de tierras tanto a nivel nacional como en el norte de La Libertad, Burke (1976) calculaba, basado en datos del Censo Agropecuario de 1971, que ya en los años sesenta el $49 \%$ de las explotaciones en todo el país era de menos de una hectárea. Esta tendencia se mantiene en la actualidad, ya que el $68 \%$ de las explotaciones agrícolas en todo el país tienen menos de 1 hectárea mientras que en el departamento de La Libertad ese porcentaje sube hasta 77\% (MINEC,2009). Por ello, se identifica que en la reestructuración de tierras dentro del sistema agrícola cafetalero surge el germen que determina la actual estructura de posesión de tierras en el país y en la zona de estudio, caracterizada por un reducido número de grandes terratenientes y un amplio número de pequeños agricultores.

Adicionalmente a esta acumulación de tierras, muchas de las irán a diversificar sus actividades económicas en bancos, bienes raíces, construcción, aseguradoras, entre otros rubros económicos. Por ejemplo, la mencionada familia Dueñas con grandes propiedades en la zona de estudio, participaba hasta mediados de la década de 2000's, en no menos de dos grandes conglomerados económicos: Conglomerado Cuscatlán, con activos de al menos 6,722 millones de dólares y del Conglomerado Banagrícola con al menos veinte empresas y activos no inferiores a 5,998 millones de dólares ${ }^{1}$ (Asociación Equipo Maíz, 2005). De la misma forma, la familia Sol forma parte del conglomerado económico Grupo Banco de Comercio, con activos no inferiores a 1,163 millones de dólares; y del Grupo Agrisal con al menos diez empresas y activos no inferiores a 520 millones de dólares (Ibid, 2005) ${ }^{2}$. Si bien la estructura de conglomerados irá a cambiar con la venta a empresas extranjeras de grandes corporaciones como bancos, aseguradoras y otros durante la década de los noventa; es evidente que mucha de la concentración de la riqueza en el país tiene como base la agricultura y los procesos de acumulación derivados de la

\footnotetext{
${ }^{1}$ Ambas cantidades en precios corrientes.

${ }^{2}$ Ambas cantidades en precios corrientes.
} 
expropiación de tierras ejidales y comunales a finales del siglo XIX. En consecuencia, parte de la pobreza del país, que según cifras oficiales alcanzó en 2014 el $31.8 \%$ de la población (DIGESTYC, 2015), no podría entenderse de forma adecuada sin ver el panorama mayor de acumulación de riqueza del siglo XIX. Autores Como Menjívar (1980), señalan que este período de finales del siglo XIX es cuando acontece en El Salvador la acumulación originaria de capital ${ }^{3}$, donde la expropiación de tierras comunales tendrá un papel absolutamente central; contrario a lo que ocurrió en países cercanos como Guatemala y México.

Por otra parte, El sistema agrario liberal ha puesto de manifiesto la actual tendencia de utilizar insumos agrícolas no orgánicos y semillas híbridas, en substitución de las semillas criollas. Esto torna a la actual agricultura salvadoreña, particularmente a la agricultura de granos básicos, altamente dependiente del exterior, ya que los mencionados insumos son generalmente importados y que los mismos representan un alto porcentaje en el costo final de la producción agrícola. Martínez (2007) calcula que el 43.3\% de los costos de producción del maíz en El Salvador corresponden a la adquisición de agroquímicos y plaguicidas. Para el caso del arroz el porcentaje sube hasta $47.1 \%$ y de $35.4 \%$ para el caso del frijol; con una tendencia parecida en algunas frutas y legumbres (Ibid, 2007). En ese sentido, el mismo Banco Central de Reserva (2008) reconocía que es previsible la volatilidad de los precios de los alimentos si acontecen subidas intensas en los precios de los insumos. Esto afectaría directamente la zona de estudio en el norte de la libertad, al ser grandes productores de maíz y granos básicos en general. Con ello, no es despreciable la posibilidad que el sistema agrario salvadoreño, y del norte de la libertad, pueda entrar en crisis si los precios de los insumos agrícolas, particularmente de los derivados del petróleo, registren aumentos constantes, estables y a largo plazo hasta alcanzar niveles prohibitivos;

\footnotetext{
3 La misma puede ser entendida en su versión más marxista como el proceso histórico de disociación entre el productor y los medios de producción. Consultar Menjivar (1980) y Montoya (1984) entre otros.
}

generando que la adquisición de los mismos sean imposibles para la mayoría de productores locales, particularmente para los productores con menos recursos económicos y con pequeñas propiedades.

\section{Consideraciones Finales}

Como ha podido quedar de manifiesto, la evolución de los sistemas agrícolas al norte de La Libertad sigue una trayectoria relativamente estable. La región ha sido originalmente productora de alimentos para autoconsumo, marcadamente granos básicos y otras hortalizas, mientras se tenían algunos otros productos particulares para su explotación, ya sea añil, café, azúcar, etc.

En el sistema agrario indígena, se puede notar un punto de quiebre cuando se pasa a domesticar y a cultivar los suelos de forma más permanente, cultivando alimentos de forma marcada para auto subsistencia y con algunas herramientas artesanales. Posteriormente, en el sistema agrario colonial, la mano de obra estará mucho más controlada, se seguirán produciendo alimentos para auto consumo y simultáneamente se producirá añil motivado por un interés mercantil de los conquistadores españoles. La conquista, además de producir dramas humanos y desaparición de poblaciones indígenas, acarrearía paralelamente un aumento en las herramientas para la utilización en agricultura, así como la introducción de ganado. En el caso del sistema agrario cafetalero del norte de la libertad, así como en todo el país, se puso de manifiesto que existe relación estrecha entre la acumulación proveniente del sector agrícola de finales del siglo XIX y acumulación de riqueza en general. En ese sentido, muchas familias que tenían el origen de su riqueza en las actividades agrícolas, lograron hacer la transición hacia otros importantes rubros de negocios. De forma similar, el enriquecimiento de un número reducido de empresarios trajo consigo el empobrecimiento de la gran mayoría campesina. El sistema agrario liberal por su vez traerá aparejado una reducción de la importancia del sector agrícola en general y una substitución en los insumos utilizados. 


\section{Referencias}

ADAMS, J. Vegetation-Climate Interaction: How Plants Make the Global Environment. Springer. Chichester. 2007.

AMBROSINI, L.; MIGUEL, L.; FILIPPI, E.. Evolução e diferenciação dos sistemas agrários nos Campos de Cima da Serra: origem dos pecuaristas familiares produtores do Queijo Serrano. Desenvolvimento e Meio Ambiente (UFPR), v. 26, p. 171-187, 2012.

ALVEAR, V. La Educación Popular en Morazán, El Salvador, durante la guerra civil de 1981 a 1992: ¿parte de una estrategia de supervivencia?. Tesis Doctoral. Doctorado en Educación. Freie Universität Berlim. Alemania. 2002.

ASOCIACIÓN EQUIPO MAÍZ. Los más ricos de El Salvador. 2 ed. San Salvador. 2005.

BANCO CENTRAL DE RESERVA -BCR-. Boletín Económico. La Economía Nacional y de los Hogares Salvadoreños: Impacto de los Precios de los Alimentos y del Petróleo. San Salvador. 2008.

SILVA NETO, B. ; BASSO, D. ; LIMA, A. P. Teoria dos Sistemas Agrários: uma nova abordagem do desenvolvimento da agricultura. Extensão Rural v. 4, p. 6-19, 1997.

BURKE, M. () El sistema de plantación y la proletarización del trabajo agrícola en El Salvador. School of Economics Faculty Scholarship. Paper 13. 1976. Acceso el 1/2/2016.

http://digitalcommons.library.umaine.edu/eco_fac pub/13

CHING, E. El levantamiento de 1932. En Herrera, S. (Coor) y Rivera, E. (2011). El Salvador: Historia Mínima. 1 Ed. San Salvador. Secretaria de la Cultura de la Presidencia de la República.2011.

Comisión Municipal de Protección Civil -CMPC-. Plan de atención a emergencias período invernal abril 2013. 2013. Consultado 1 de Julio de 2015. www.proteccioncivil.gob.sv/zonadescargas/plane s muncipales/05. La Libertad Ok/Plan invernal munic LaLibertad 2 013.pdf.

COOPERACIÓN TÉCNICA FRANCESA (CTF). Estudio de un sistema agrario: El distrito de riego y avenimiento de Zapotitán. Realidad: Revista de Ciencias Sociales y Humanidades. Año 1, No. 5. Septiembre - Octubre. P 413-451. 1988.

COOPERACIÓN TÉCNICA FRANCESA (CTF). Propuesta metodológica para el análisis de una realidad agraria. Realidad: Revista de Ciencias
Sociales y Humanidades. Año 1, No. 2. Marzo Abril. P 107-148. 1988b.

COTRIM, D. ; MIGUEL, L. Renda da pesca artesanal: análise dos sistemas de produção na pesca em Tramandaí ? RS. Redes (Santa Cruz do Sul), v. 14, p. 5-23, 2009.

DIRECCIÓN GENERAL DE ESTADÍSTICA Y CENSOS -DIGESTYC-. Encuesta de Hogares y Propósitos Múltiples 2014. San Salvador. 2015.

DIXON, C. Rethinking southeast Maya agriculture: A view from the manioc fields of Joya de Cerén, El Salvador. La Universidad: Nueva Época. San Salvador. Núm. 14. P215-228. 2011.

Fundación Salvadoreña para la reconstrucción y el desarrollo -REDES- La agricultura familiar en el centro de las políticas agrícolas. Análisis del Plan de Agricultura Familiar (PAF) y su presupuesto. El Salvador, 2011-2014. San Salvador. 2014

KRAEMER, M.F.; MIGUEL, L. Transformações históricas e diferenciação dos sistemas agrários: as estratégias de reprodução socioeconômica entre as famílias quilombolas de Morro Alto (Maquiné - RS - Brasil). Redes (Santa Cruz do Sul. Online), v. 19, p. 258-285, 2014.

LAURIA, A. An Agrarian Republic Commercial Agriculture and the Politics of Peasant Communities in El Salvador, 1823-1914. Estados Unidos. University of Pittsburgh Press. 1999.

LINDO, H. Tierra, economía y sociedad en el siglo XIX. En Herrera, S. (Coor) y Rivera, E. (2011). El Salvador: Historia Mínima. 1 Ed. San Salvador. Secretaria de la Cultura de la Presidencia de la República. 2011.

MARTÍNEZ, G.Implicaciones de las normas de propiedad intelectual contenidas en el CAFTADR con relación al mercado de agroquímicos. San Salvador. Funde. 2007.

MARTÍNEZ, O. (Coord). El Salvador. Historia General. 1 ed. San Salvador. Editorial Nuevo Enfoque. 2002.

MAYOZER, M. y ROUDART, L. Historie des agricultures du monde: du néolitique à la crise contemporaine. Paris. Seuil. 1997.

MAYOZER, M. y ROUDART, L. História das agriculturas no mundo Do neolítico à crise contemporânea. 1 Ed. Brasil. UNESP. 2010.

MCKEE, B. Structure 7 excavations. En SHEETS, P; MCKEE, B. (eds). 1990 Research at the Cerén site, El Salvador: A preliminary report. Boulder. Department of Anthropology. University of Colorado, Boulder. 1990. 
MCKEE, B. Archaeological Investigations in Operation 2, Joya de Cerén. En SHEETS, P.; BROWN, L.(Eds). Preliminary Report of the Cerén Research Project 1996 Field Season. Boulder. Department of Antropology. University of Colorado. 1996.

MENJIVAR, R. Acumulación originaria y desarrollo del capitalismo en El Salvador. San Salvador. 1 Ed. Editorial Universitaria Centroamericana EDUCA. 1980.

MIGUEL, L. Evolução e diferenciação de sistemas agrários, desenvolvimento rural e a história da agricultura no Brasil. Em ALMEIDA, J. (2009). Políticas Públicas e desenvolvimento rural: Percepções e perspectivas no Brasil e em Moçambique. Porto Alegre, Brasil. UFRGS. 2009.

MILLER, D. Volcanology, stratigraphy, and effects on structures. In SHEETS, P. Before the volcano erupted. The ancient Cerén Village in Central America. Parte I, NO. 2. Sin número. University of Texas Press Austin. 1 Ed. Austin. 2002.

MINISTERIO DE AGRICULTURA Y GANADERÍA MAG- (2014). Anuario de Estadísticas Agropecuarias (DEA). Encuesta Nacional Agropecuaria de Propósitos Múltiples 2013-2014 (ENAPM 2013-2014). Precios de Mercado. 2014. Consultado $1 / 7 / 2015$ http://www.mag.gob.sv/index.php?option=com p hocadownload \&view=category\&download=1408: anuario\&id=14:anuariosagropecuarios\&ltemid $=224$

MINISTERIO DE AGRICULTURA Y GANADERÍA MAG-. Caracterización de la cadena productiva de maíz blanco en El Salvador. IICA, CENTA, MAG. Santa Tecla, s.d.

MINISTERIO DE ECONOMIA -MINEC-. VI Censo de Población y Vivienda 2007. Dirección General de Estadísticas y Censos. 2008.

MINISTERIO DE ECONOMÍA -MINEC-. IV Censo Agropecuario 2007-2008. Resultados Nacionales. San Salvador. 2009.

MINISTERIO DE EDUCACIÓN -MINED-. Historia 1 de El Salvador. 2 Edición. San Salvador. 2008.

MINISTERIO DE EDUCACIÓN -MINED-. Historia 2 de El Salvador. 2 Edición. San Salvador. 2008b.

MINISTERIO DE MEDIO AMBIENTE Y RECURSOS NATURALES -MARN- (2015). Boletín Climático Anual 2014. Consultado: 1/7/2015. http://mapas.snet.gob.sv/meteorologia/climatico2 014.pdf.

MONTOYA, A. Acerca del problema de la acumulación originaria. Boletín de Ciencias Económicas y Sociales. Año VII. No. 1. P 41-48. 1984.
MORLEY, S. y BRAINERD, G. The Ancient Maya. $4^{\circ}$ Ed. California. Standford University Press. 1983.

ORGANIZACIÓN DE ESTADOS AMERICANOS OEA-. El Salvador - Zonificación Agrícola - Fase II - Sistema de Información para el Desarrollo. Secretaría General. Washington D.C. Estados Unidos. 1977.

PORTILLO, G. El origen de la burguesía cafetalera: Caso la libertad. En MARTINEZ, O. (Coord). EI Salvador: Historia General. $1^{\circ}$ Ed. San Salvador. Editorial Nuevo Enfoque. 2002.

PROGRAMA SALVADOREÑO DE INVESTIGACIÓN SOBRE DESARROLLO Y MEDIO AMBIENTE PRISMA- (2002). Cambio económico, empleo y pobreza rural en El Salvador. Documento de Trabajo. Consultado 1/7/2015. http://www.prisma.org.sv/uploads/media/Cambio economico.pdf.

RIBAS, R.; MIGUEL, L.. A herança sesmeirapecuarista e seus reflexos sobre o desenvolvimento atual: o caso de Encruzilhada do Sul - RS, segundo uma abordagem de sistemas agrários. História Econômica \& História de Empresas, São Paulo, v. 3, n.1, p. 1-20, 2005.

ROSA, H. Transformación Económica y Medio Ambiente: De las Exportaciones Agrícolas a la Exportación de Mano de Obra. En BLANCO, $\mathrm{H}$. TOGEIRO, L. y

SHEETS, P. . Dwelling in the Ancestral Joya de Cerén Village.En Rosemary, J. (2013). Revelaing Ancestral Central America. 1 Ed. Washington, D.C. P. 23-32. Smithsonian Institution. 2013.

SHEETS, P.; DIXON, C.; GUERRA, M.; BRANFORD, A. Manioc Cultivation at Cerén, El Salvador: Occasional Kitchen Garden Plant or Staple Crop?" Ancient Mesoamerica. Vol. 22. P 1-11. 2011.

SHEETS,P.; LENTZ, D.;PIPERNO, D.;JONES, J.;DIXON, C.; MALOOF, G.; HOOD, A. Ancient manioc agriculture south of the cerenvillage, el Salvador. Latin American Antiquity. Vol 23(3).pp. 259-281. 2012.

SHEETS, P.; SHEEHAN, A.; DIXON, C.; TETREAULT, J. SÉLLER, E. En búsqueda del límite occidental del sitio arqueológico Joya de Cerén. El Salvador Investiga. Año 2, No. 4. P 3438. 2006.

SOLÓRZANO, J.C. Pueblos de indios y explotación en la Guatemala y El Salvador coloniales. Anuario de Estudios Centroamericanos. Vol 8. P. 125-133. 1982.

UNITED STATES AGENCY FOR INTERNATIONAL DEVELOPMENT -USAID-. Agricultural supply marketing system in El Salvador. Washington, Estados Unidos. 1975. 
UNITED STATES AGENCY INTERNATIONAL DEVELOPMENT -USAID- (2010). Informe sobre los bosques tropicales y biodiversidad en El Salvador. Accesado: 1/7/2015. http://www.corfores.com/library/645625323_2013 0301014321.pdf

WHITE, C. The History of El Salvador (The Greenwood Histories of the Modern Nations). Estados Unidos. Greenwood Press. 2008. Retrieved 7 August 2015, from http://www. myilibrary.com?ID=242066.

WORLD BANK. (2015). Promedio detallado de precipitaciones ( $\mathrm{mm}$ anuales). 2015. Acceso 1/2/2016.

Sitio: http://datos.bancomundial.org/indicador/AG.LND. PRCP.MM 